TRANSACTIONS OF THE

AMERICAN MATHEMATICAL SOCIETY

Volume 350, Number 11, November 1998, Pages 4461-4472

S $0002-9947(98) 02291-0$

\title{
ON THE ZEROS OF A POLYNOMIAL AND ITS DERIVATIVES
}

\author{
PIOTR PAWLOWSKI
}

\begin{abstract}
If $p(z)$ is univariate polynomial with complex coefficients having all its zeros inside the closed unit disk, then the Gauss-Lucas theorem states that all zeros of $p^{\prime}(z)$ lie in the same disk. We study the following question: what is the maximum distance from the arithmetic mean of all zeros of $p(z)$ to a nearest zero of $p^{\prime}(z)$ ? We obtain bounds for this distance depending on degree. We also show that this distance is equal to $\frac{1}{3}$ for polynomials of degree 3 and polynomials with real zeros.
\end{abstract}

\section{INTRODUCTION}

Let $\mathcal{P}_{n}$ denote the family of all complex polynomials of exact degree $n$, having all zeros in the closed unit disk, i.e.

$$
\mathcal{P}_{n}:=\left\{p(z)=a_{n} \prod_{k=1}^{n}\left(z-z_{k}\right), \quad\left|z_{k}\right| \leq 1 \quad\left(1 \leq k \leq n, a_{n} \neq 0\right)\right\}
$$

and let

$$
\mathcal{P}:=\bigcup_{n \geq 1} \mathcal{P}_{n}
$$

Let $p \in \mathcal{P}$. From the Gauss-Lucas theorem we know that $p^{\prime} \in \mathcal{P}$. A well-known attempt to further characterize the location of the zeros of $p^{\prime}$, which are also called the critical points of $p$, is the Sendov Conjecture.

Conjecture 1.1 (Sendov). If $p(z) \in \mathcal{P}$ then each of the disks $z \in \mathbb{C}:\left|z-z_{k}\right| \leq 1$, $k=1, \ldots, n$, contains at least one zero of $p^{\prime}(z)$.

The Sendov Conjecture has been verified in a number of special cases [2]. Let $H(p)$ denote the convex hull of the zeros of a polynomial $p$. Schmeisser [10] posed the following generalization of the Sendov Conjecture.

Conjecture 1.2 (Schmeisser). If $p(z) \in \mathcal{P}$, then for every $\zeta \in H(p)$, the disk $\{z:|z-\zeta| \leq 1\}, k=1, \ldots, n$, contains at least one zero of $p^{\prime}(z)$.

Schmeisser [10] showed that this conjecture is true if $H(p)$ is a triangular region. It is also shown to be true if $H(p)$ has all its vertices on the unit circle [2]. We investigate a special case of the Schmeisser problem: we concentrate on one point in $H(p)$ - the average of zeros of $p$. Although in this case the Schmeisser Conjecture follows immediately from the Gauss-Lucas Theorem, we show that the disk $\{z$ :

Received by the editors June 27, 1996.

1991 Mathematics Subject Classification. Primary 30C15.

Key words and phrases. Polynomials, location of zeros.

(C)1998 American Mathematical Society 
$|z-\zeta| \leq 1\}$ in Conjecture (1.2) can be replaced by a smaller concentric disk with radius $r$. We give bounds for $r$ depending on $n$ for any $p \in \mathcal{P}_{n}$. We also obtain sharp estimates of $r$ for polynomials of degree 3 and for polynomials having only real zeros.

Let

$$
p(z)=\sum_{k=0}^{n} a_{k} z^{k}=a_{n} \prod_{k=1}^{n}\left(z-z_{k}\right) \in \mathcal{P}_{n} .
$$

Then the centroid, $A_{p}$, of the polynomial $p(z)$ is the arithmetic average of its zeros:

$$
A_{p}:=\frac{1}{n}\left(z_{1}+\cdots+z_{n}\right)=-\frac{1}{n} \frac{a_{n-1}}{a_{n}} .
$$

It is easy to check that the centroid is differentiation invariant. Namely, for $n>1$

$$
p^{\prime}(z)=\sum_{k=0}^{n-1}(k+1) a_{k+1} z^{k}=n a_{n} \prod_{k=1}^{n-1}\left(z-\zeta_{k}\right),
$$

so that

$$
A_{p^{\prime}}=\frac{1}{n-1}\left(\zeta_{1}+\cdots+\zeta_{n-1}\right)=-\frac{1}{n-1} \frac{(n-1) a_{n-1}}{n a_{n}}=-\frac{1}{n} \frac{a_{n-1}}{a_{n}}=A_{p},
$$

which gives

$$
A_{p}=A_{p^{(k)}}, \quad 0 \leq k \leq n-1 .
$$

We also define

$$
J(p):=\min _{1 \leq k \leq n-1}\left|A_{p}-\zeta_{k}\right| \text { and } \gamma_{n}:=\sup _{p \in \mathcal{P}_{n}} J(p) .
$$

Remark 1.3. There exist results that characterize the location of zeros of $p^{(k)}$ for $k \geq 1$ for polynomials having only real zeros. Milovanovic et al. [6] give an account of many results of this type. For instance, if $p$ is a polynomial with zeros $x_{1} \leq x_{2} \leq \cdots \leq x_{n}$, then we define $\operatorname{span}(p):=x_{n}-x_{1}$. Clearly,

$$
\operatorname{span}\left(p^{(k)}\right) \leq \operatorname{span}(p), \quad 0 \leq k \leq n-1 .
$$

by the Gauss-Lucas Theorem. Robinson [8], [9] has shown that the strict inequality holds in (1.9) if $\mathrm{k}$ is sufficiently large. He also gave estimates on $\operatorname{span}\left(p^{(k)}\right)$, depending on $\operatorname{span}(p), n$ and $k$. Since the centroid of a polynomial is invariant under differentiation, the zeros of $p^{(k)}$ must be getting closer to $A_{p}$ as $k$ grows big. Although $\operatorname{span}\left(p^{(k)}\right)$ may not decrease if $k<n / 2$, we will show in section 4 , that not all zeros of $p^{\prime}$ may stay away from $A_{p}$.

\section{A LOWER BOUND FOR THE CONSTANT $\gamma_{n}$}

We first give simple upper and lower bounds for $\gamma_{n}$.

\section{Proposition 2.1.}

$$
\text { For any } n>1, n^{-\frac{1}{n-1}} \leq \gamma_{n} \leq 1
$$

Proof. The second inequality follows from the fact that the centroid of a polynomial is differentiation invariant, so the centroid of $p(z)$ is also the centroid of $p^{\prime}(z)$. Since all of the zeros of $p^{\prime}(z)$ are inside the unit circle, at least one of them lies within distance 1 from the centroid. The first inequality follows from the special choice of 
$p(z)=z^{n}-z$. Here, we have $p^{\prime}(z)=n z^{n-1}-1$ and for every critical point $\zeta_{k}$ of $p(z)$, we have

$$
\left|\zeta_{k}\right|=\frac{1}{n^{\frac{1}{n-1}}} \text { so } J(p)=\frac{1}{n^{\frac{1}{n-1}}} .
$$

Remark 2.2. $1-n^{-\frac{1}{n-1}}$ is asymptotically equal to $\frac{\log n}{n}$, i.e.

$$
\lim _{n \rightarrow \infty} \frac{1-n^{-\frac{1}{n-1}}}{\frac{\log n}{n}}=1 .
$$

We can show it by taking Taylor series expansion of $1-n^{-\frac{1}{n-1}}$ :

$$
\begin{aligned}
1-n^{-\frac{1}{n-1}} & =1-\exp \left(-\frac{\log n}{n-1}\right)=\frac{\log n}{n-1}-\frac{1}{2 !}\left(\frac{\log n}{n-1}\right)^{2}+\frac{1}{3 !}\left(\frac{\log n}{n-1}\right)^{3}-\ldots \\
& =\frac{\log n}{n-1}+o\left(\frac{\log n}{n-1}\right) .
\end{aligned}
$$

Therefore, there exist constants $c_{1}, c_{2}$ such that

$$
1-c_{1} \frac{\log n}{n}<n^{-\frac{1}{n-1}}<1-c_{2} \frac{\log n}{n} .
$$

\section{AN UPPER BOUND FOR CONSTANT $\gamma_{n}$}

If $p$ is monic and $A_{p}=0$, the product of all critical points is $\prod_{k=1}^{n-1} \zeta_{k}=\left|a_{1}\right| / n$, so $J(p)$ is bounded by $\sqrt[n-1]{\left|a_{1}\right| / n}$. We can use a good upper bound on $\left|a_{1}\right|$ given by the following lemma:

\section{Lemma 3.1.}

$$
\text { If } p(z)=z^{n}+\cdots+a_{1} z+a_{0} \in \mathcal{P}_{n} \text { and } A_{p}=0, \text { then }\left|a_{1}\right|=\left|p^{\prime}(0)\right| \leq 1 .
$$

Proof. If $A_{p}=0$, then

$$
\begin{aligned}
\left|a_{1}\right| & =\left|\sum_{k=1}^{n} \prod_{j \neq k} z_{j}\right|=\left|\sum_{k=1}^{n}\left(\prod_{j \neq k} z_{j}-\overline{z_{k}} \prod_{j=1}^{n} z_{j}\right)\right| \\
& =\left|\sum_{k=1}^{n}\left(1-\left|z_{k}\right|^{2}\right) \prod_{j \neq k} z_{j}\right| \leq \sum_{k=1}^{n}\left(1-\left|z_{k}\right|^{2}\right) \prod_{j \neq k}\left|z_{j}\right| .
\end{aligned}
$$

Let $\left|z_{k}\right|=r_{k}, 0 \leq r_{k} \leq 1,1 \leq k \leq n$. and let

$$
B_{n}:=\sum_{k=1}^{n}\left(1-r_{k}^{2}\right) \prod_{j \neq k} r_{j}, \quad n \geq 1 .
$$

We will show that

$$
B_{n} \leq 1 \text { for every } n \geq 1 \text {. }
$$

First, we will show that $B_{n}$ attains its maximum on the boundary of the set $[0,1]^{n}$. If $n=1$, then the maximum of $B_{1}=1-r_{1}$ is attained at $r_{1}=0$. If $n \geq 2$ and $B_{n}$ 
attains its maximum on the set $[0,1]^{n}$ at some point $\left(r_{1}, \ldots, r_{n}\right) \in(0,1)^{n}$. Then we have

$$
\frac{\partial B_{n}}{\partial r_{k}}=-2 r_{k} \prod_{j \neq k} r_{j}+\sum_{j \neq k}\left(1-r_{j}^{2}\right) \prod_{i \neq k, j} r_{i}=0, \quad 1 \leq k \leq n .
$$

Then

$$
\text { (3.6) } \begin{aligned}
r_{k} \frac{\partial B_{n}}{\partial r_{k}} & =-2 r_{k}^{2} \prod_{j \neq k} r_{j}+\sum_{j \neq k}\left(1-r_{j}^{2}\right) \prod_{i \neq j} r_{i} \\
& =\left(-1-r_{k}^{2}\right) \prod_{j \neq k} r_{j}+\sum_{j=1}^{n}\left(1-r_{j}^{2}\right) \prod_{i \neq j} r_{i} \\
& =\left(-r_{k}-\frac{1}{r_{k}}\right) \prod_{j=1}^{n} r_{j}+\sum_{j=1}^{n}\left(1-r_{j}^{2}\right) \prod_{i \neq j} r_{i}=0, \quad 1 \leq k \leq n .
\end{aligned}
$$

For $i \neq j$ we get

$$
r_{i} \frac{\partial B_{n}}{\partial r_{i}}-r_{j} \frac{\partial B_{n}}{\partial r_{j}}=\left(r_{j}+\frac{1}{r_{j}}-r_{i}-\frac{1}{r_{i}}\right) \prod_{k=1}^{n} r_{k}=0 .
$$

Hence

$$
r_{j}+\frac{1}{r_{j}}=r_{i}+\frac{1}{r_{i}}
$$

Since the function $f(x)=x+\frac{1}{x}$ is one to one on $(0,1), r_{i}=r_{j}$. Thus the local maximum $M$ of $B_{n}$ can be attained at $\left(r_{1}, \ldots, r_{n}\right) \in(0,1)^{n}$ only if all $r_{k}$ are equal. Let $r:=r_{1}=\cdots=r_{n}$. Then

$$
M \leq \sup _{0<r<1}\left(n r^{n-1}\left(1-r^{2}\right)\right) .
$$

The above supremum is attained at $r=\sqrt{\frac{n-1}{n+1}}$ and

$$
M \leq \frac{2 n}{n+1}\left(1+\frac{2}{n-1}\right)^{-\frac{n-1}{2}}, \quad n \geq 2 .
$$

It is well known that the sequence $e_{n}:=\left(1+\frac{2}{n-1}\right)^{-\frac{n-1}{2}} \rightarrow \frac{1}{e}$ as $n \rightarrow \infty$ and $e_{n}$ is decreasing. Thus, for $n \geq 3, e_{n} \leq e_{3}=1 / 2$. Therefore, from (3.10) we have

$$
M \leq \begin{cases}\frac{4}{3 \sqrt{3}}<1 & \text { if } n=2 \\ \frac{n}{n+1}<1 & \text { if } n \geq 3 .\end{cases}
$$

Since $B_{n}=1$ if $\left(r_{1}, \ldots, r_{n}\right)=(0,1, \ldots, 1), B_{n}$ must attain its maximum on the boundary of $[0,1]^{n}$.

Now, we will prove (3.4) by induction. If $n=1$, then $B_{n}=1-r_{1} \leq 1$. Let $N$ be fixed and assume that (3.4) holds for $n=1, \ldots, N-1$. If $B_{N}$ attains its maximum on the set $[0,1]^{n}$ at the point $\left(r_{1}, \ldots, r_{n}\right)$, then $r_{k}=0$ or $r_{k}=1$ for some $k, 1 \leq k \leq N$. If $r_{k}=0$, then $B_{N}=\prod_{j \neq k} r_{j} \leq 1$. Otherwise, if $r_{k}=1$, then $B_{N}=B_{N-1} \leq 1$ by induction.

Remark 3.2. From Lemma 3.1 we get an estimate on $J(p)$ in the special case when $A_{p}=0$. Then we have $J(p) \leq\left(\left|p^{\prime}(0)\right| / n\right)^{\frac{1}{n-1}} \leq(1 / n)^{\frac{1}{n-1}}$. This estimate is sharp as shown by $p(z)=z^{n}-z$. 
Using Lemma 3.1 we can find an upper bound on $\gamma_{n}$ that is smaller than 1 .

\section{Theorem 3.3.}

$$
\gamma_{n} \leq \frac{2 n^{\frac{1}{n-1}}}{n^{\frac{2}{n+1}}+1} .
$$

Proof. If $p(z) \in \mathcal{P}_{n}$ and $\left|A_{p}\right|=r$, then

$$
q(z)=\frac{1}{(r+1)^{n}} p\left(A_{p}+(r+1) z\right) \in \mathcal{P}_{n} \text { and } A_{q}=0 .
$$

The polynomial $q(z)$ is monic, so by Lemma 3.1, there exist $w$ such that $q^{\prime}(w)=0$ and

$$
|w| \leq\left(\frac{\left|q^{\prime}(0)\right|}{n}\right)^{\frac{1}{n-1}} \leq \frac{1}{n^{\frac{1}{n-1}}} .
$$

If $\xi=(r+1) w+A_{p}$, then $p^{\prime}(\xi)=0$ and

$$
J(p) \leq\left|\xi-A_{p}\right|=(r+1)|w| \leq \frac{r+1}{n^{\frac{1}{n-1}}} .
$$

On the other hand, there must be a zero of $p^{\prime}(z)$ in the half-plane $\operatorname{Re}\left(z \overline{A_{p}}\right) \geq r$, so we have

$$
J(p) \leq \sqrt{1-r^{2}}
$$

Therefore, from (3.15) and (3.16)

$$
J(p) \leq \sup _{0 \leq r \leq 1} \min \left\{\frac{r+1}{n^{\frac{1}{n-1}}}, \sqrt{1-r^{2}}\right\} .
$$

As functions of $r$, the bounds in (3.15) and (3.16) are respectively increasing and decreasing on the interval $[0,1]$, so the supremum in (3.17) is attained if $r$ is the unique solution of the equation

$$
\frac{r+1}{n^{\frac{1}{n-1}}}=\sqrt{1-r^{2}},
$$

which gives

$$
r=\frac{n^{\frac{2}{n-1}}-1}{n^{\frac{2}{n-1}}+1} \text { and } J(p) \leq \frac{2 n^{\frac{1}{n-1}}}{n^{\frac{2}{n-1}}+1} .
$$

Remark 3.4. Theorem 1.1 leads to the following estimate

$$
\gamma_{n} \leq \frac{2 n^{\frac{1}{n-1}}}{n^{\frac{2}{n-1}}+1}=1-\frac{\left(1-n^{\frac{1}{n-1}}\right)^{2}}{n^{\frac{2}{n-1}}+1}<1-c\left(\frac{\log n}{n}\right)^{2},
$$

where $c$ is some constant, because as we have already noted in (2.3) $1-n^{-\frac{1}{n-1}}$ is asymptotically equal to $\log n / n$. However, the estimate (3.20) doesn't seem to be sharp. Our conjecture is that a sharp inequality would have the form $\gamma_{n} \leq 1-c \frac{\log n}{n}$, as in the example $p(z)=z^{n}-z$. 


\section{Extremal polynomials of LOW DEGREeS}

In this section we compute $\gamma_{3}$ exactly. In order to do this we restrict our attention to extremal polynomials, i.e. the subset of $\mathcal{P}_{n}$ consisting of polynomials for which $J(p)=\gamma_{n}$. Such polynomials exist since $J(p)$ is continuous in the roots of $p^{\prime}$, which are continuous in the coefficients of $p$, which are continuous in the roots of $p$ (being elementary symmetric functions of those roots). The roots of $p$ are contained in the compact set $D$, so $J(p)$ achieves its supremum on $D$. For extremal problems of this type, Phelps and Rodriguez ([7]) have shown that every extremal polynomial has at least 2 zeros on the unit circle and that every sub-arc of the unit circle of length at least $\pi$ must contain at least one zero. Otherwise, it would be possible to inscribe all zeros of the polynomial in the circle with radius smaller than 1 , which would contradict extremality. We can conclude that unless the extremal polynomial has 2 zeros at the endpoints of diameter of the unit circle, it must have at least 3 zeros on the unit circle. This is also valid in our problem.

Proposition 4.1. $\gamma_{3}=\frac{2}{3}$, and all extremal polynomials are of the form $p(z)=$ $q\left(e^{i t} z\right)$ where $q(z)=z^{3}-z^{2}-z+1$

Proof. Let $p(z) \in \mathcal{P}_{3}$. Without loss of generality we can assume that 1 is one of the zeros of $p(z)$, i.e.

$$
p(z)=(z-1)\left(z-z_{1}\right)\left(z-z_{2}\right)
$$

and

$$
A_{p}=\frac{1+z_{1}+z_{2}}{3}
$$

Since $p^{\prime}(z) / 3$ is monic, the distance between $A_{p}$ and the nearest critical point is bounded by $\sqrt{\left|p^{\prime}\left(A_{p}\right) / 3\right|}$. We have

$$
\frac{\left|p^{\prime}\left(A_{p}\right)\right|}{3}=\frac{1}{9}\left|-z_{1}^{2}-z_{2}^{2}+z_{1}+z_{2}+z_{1} z_{2}-1\right| .
$$

By the maximum modulus principle $\left|p^{\prime}\left(A_{p}\right) / 3\right|$ attains its maximum on $D$ when both $z_{1}$ and $z_{2}$ are on the unit circle. We can also assume that $p(z)$ is extremal and $z_{1}=e^{i \phi_{1}}, 0 \leq \phi_{1} \leq \pi$, and $z_{2}=e^{i \phi_{2}}, \pi \leq \phi_{2} \leq 2 \pi$, and $\phi_{2}-\phi_{1} \leq \pi$. Then

$$
\begin{aligned}
\frac{\left|p^{\prime}\left(A_{p}\right)\right|}{3} & \leq \frac{1}{9}\left(\left|z_{1}\right|^{2}+\left|z_{2}\right|^{2}+\left|z_{1}+z_{2}+z_{1} z_{2}-1\right|\right) \\
& \leq \frac{2}{9}+\frac{1}{9} \sqrt{4+4 \sin \phi_{1} \sin \phi_{2}} \leq \frac{4}{9}
\end{aligned}
$$

where the last inequality follows from the fact that $\sin \phi_{1} \sin \phi_{2} \leq 0$. Thus we have

$$
J(p) \leq \sqrt{\frac{4}{9}}=\frac{2}{3},
$$

with equality only if $\sin \phi_{1} \sin \phi_{2}=0$. In this case either $z_{1}=z_{2}=-1$ and $p(z)=$ $(z+1)^{2}(z-1)$ or $z_{1}=1, z_{2}=-1$ and $p(z)=(z-1)^{2}(z+1)$. These are the only extremal polynomials for which $p(1)=0$. In both cases $J(p)=\frac{2}{3}$, so $\gamma_{3}=\frac{2}{3}$.

Remark 4.2. Numerical computations using nonlinear optimization algorithms suggest that $\gamma_{4}=2 / 3$ and an example of extremal polynomial

$$
p_{4}(z)=z^{4}-\frac{4}{3} z^{3}+\frac{2}{3} z^{2}-\frac{4}{3} z+1 \text {. }
$$


Zeros of $p_{4}$ are

$$
z_{1}=z_{2}=1, z_{3}=-1 / 3+\frac{2 \sqrt{2}}{3} i \text { and } z_{4}=-1 / 3-\frac{2 \sqrt{2}}{3} i .
$$

Zeros of $p_{4}^{\prime}$ are

$$
w_{1}=1, w_{2}=\frac{\sqrt{3}}{3} i \text { and } w_{3}=-\frac{\sqrt{3}}{3} i .
$$

$\gamma_{5}=3 / 4$ and an example of extremal polynomial is

$$
p_{5}(z)=z^{5}-\frac{5}{4} z^{4}-\frac{5}{4} z^{3}+\frac{5}{4} z^{2}+\frac{5}{4} z+1 .
$$

Zeros of $p_{5}$ are

$$
z_{1}=z_{2}=z_{3}=1, z_{4}=-\frac{7}{8}+\frac{\sqrt{15}}{8} i \text { and } z_{5}=-\frac{7}{8}-\frac{\sqrt{15}}{8} i .
$$

Zeros of $p_{5}^{\prime}$ are

$$
w_{1}=w_{2}=1 \text { and } w_{3}=w_{4}=-1 / 2 .
$$

Both the Sendov Conjecture and our problem are special cases of extremal problems considered by Miller ([4],[5]). He defined $S(n, \beta)$ to be the set of complex polynomials of degree $n$ that have all their zeros in the unit disk and at least one zero at $\beta$ and $|p|_{\alpha}$ to be the distance between $\alpha$ and the closest root of $p^{\prime}$. Polynomial $p \in S(n, \beta)$ is said to be maximal with respect to $\alpha$ if for every $q \in S(n, \beta)$ we have $|p|_{\alpha} \geq|q|_{\alpha}$. He also defined critical circle to be the circle with center $\alpha$ and radius $|p|_{\alpha}$. Our problem can be formulated as follows: find maximum of $|p|_{A_{p}}$ for $p \in S(n, \beta)$. Miller ([4]) made the following conjecture:

Conjecture 4.3 (Miller). Let $p \in S(n, \beta)$ be maximal with respect to $\alpha$, let $s$ be the number of zeros of $p$ on the unit circle, and let $r$ be the number of zeros of $p^{\prime}$ on the critical circle. Then

$$
r=n-1, \text { and }
$$

given that all zeros of $p^{\prime}$ lie on a circle, $s$ is as large as possible

He also proved that under the hypothesis of Conjecture 1.3, $2 r+s \geq n+1$. In our problem, if $n=3,4$ or 5 , it seems that all zeros of a maximal polynomial $p$ lie on the unit circle and all zeros of $p^{\prime}$ lie on the critical circle. $p \in S(n, \beta)$ is said to be maximal with respect to $\alpha$ if for every $q \in S(n, \beta)$ we have $|p|_{\alpha} \geq|q|_{\alpha}$. He also defined critical circle to be the circle with center $\alpha$ and radius $|p|_{\alpha}$. Our problem can be formulated as follows:

\section{Polynomials with Real zeros}

The polynomial $p(z)=z^{n}-z$ with high $J(p)$ has all its zeros, except one, spread evenly around the unit circle. If this situation cannot happen, for instance for polynomials with real zeros, we may expect $J(p)$ to be lower. Let's consider the subclass of $\mathcal{P}_{n}$ consisting of polynomials having only real zeros. In this case we get the following theorem:

Theorem 5.1. If $p(x) \in \mathcal{P}_{n}$ is a polynomial having only real zeros, then

$$
J(p) \leq \frac{2}{3} .
$$


Equality in (5.1) is attained if and only if $n$ is a multiplicity of 3 and $p(x)=(x-1)^{\frac{2 n}{3}}$ $(x+1)^{\frac{n}{3}}$ or $p(x)=(x-1)^{\frac{n}{3}}(x+1)^{\frac{2 n}{3}}$

Proof. It's enough to show that (5.1) is true if $A_{p}$ is non-negative; otherwise we can consider $p(-x)$ instead of $p(x)$. If $A_{p}>1 / 3$, then obviously $J(p)<2 / 3$, so let us assume that $A_{p} \in[0,1 / 3]$. Let $I=\left(A_{p}-2 / 3, A_{p}+2 / 3\right)$. We want to show that the interval $\bar{I} \subset[-1,1]$ contains at least one zero of $p^{\prime}(x)$. By Rolle's Theorem, this is true if $I$ contains at least two zeros of $p(x)$, counting multiplicities. Let us consider the remaining two cases.

Case 1: $I$ doesn't contain any zeros of $p(x)$. Let $A_{p} \in[0,1 / 3]$ be fixed and let $n_{1}$ and $n_{2}=n-n_{1}$ be the numbers of zeros of $p(x)$ in the intervals $I_{1}=\left[-1, A_{p}-2 / 3\right]$ and $I_{2}=\left[A_{p}+2 / 3,1\right]$ respectively. The condition $x_{1}+\cdots+x_{n}=n A_{p}$ imposes some constraints on $n_{1}$ and $n_{2}$. Namely, $n_{1}$ is maximal and $n_{2}$ is minimal if we move all zeros to the right as far as possible. In the extreme situation we would have $n_{1}$ zeros at $A_{p}-2 / 3$ and $n_{2}$ zeros at 1 . We then have a linear system

$$
\left\{\begin{aligned}
n_{1}\left(A_{p}-2 / 3\right)+n_{2} & =n A_{p}, \\
n_{1}+n_{2} & =n .
\end{aligned}\right.
$$

Although the solution of (5.2) is not a pair of integers in general, they give an upper bound for $n_{1}$ and a lower bound for $n_{2}$. Similarly, if we put $n_{1}$ zeros at -1 and $n_{2}$ zeros at $A_{p}+2 / 3$ we get a linear system

$$
\left\{\begin{aligned}
-n_{1}+n_{2}\left(A_{p}+2 / 3\right) & =n A_{p}, \\
n_{1}+n_{2} & =n
\end{aligned}\right.
$$

that gives a lower bound for $n_{1}$ and an upper bound for $n_{2}$. Combining (5.2) and (5.3) we obtain range of $n_{1}$ and $n_{2}$ depending on $A_{p}$

$$
\begin{aligned}
& \frac{2 n}{5+3 A_{p}} \leq n_{1} \leq \frac{3 n\left(1-A_{p}\right)}{5-3 A_{p}}, \\
& \frac{2 n}{5-3 A_{p}} \leq n_{2} \leq \frac{3 n\left(1+A_{p}\right)}{5+3 A_{p}} .
\end{aligned}
$$

Since all zeros of $p(x)$ lie in 2 disjoint intervals, we can apply Walsh's two circle theorem ([2, p. 89]).

Theorem 5.2 (Walsh). If all the zeros of an $n_{1}$-degree polynomial $f_{1}(z)$ are contained in the closed interior of the circle $C_{1}$ with center $c_{1}$ and radius $r_{1}$ and all the zeros of an $n_{2}$-degree polynomial $f_{2}(z)$ are contained in the closed interior of the circle $C_{2}$ with center $c_{2}$ and radius $r_{2}$, then all zeros of the derivative of the product $f(z)=f_{1}(z) f_{2}(z)$ are contained in the union of $C_{1}$, if $n_{1}>1$, and $C_{2}$, if $n_{2}>1$, and a third circle $C$ with center $c$ and radius $r$ where

$$
c=\frac{n_{1} c_{2}+n_{2} c_{1}}{n_{1}+n_{2}} \text { and } r=\frac{n_{1} r_{2}+n_{2} r_{1}}{n_{1}+n_{2}} .
$$

Moreover, if the closed interiors of the circles $C_{1}, C_{2}$ and $C$ have no point in common, the number of zeros of $f^{\prime}(z)$ which they contain is respectively $n_{1}-1, n_{2}-1$ and 1.

By (5.6), all zeros of $p^{\prime}(x)$ are contained in the union of the intervals $I_{1}, I_{2}$ and $I_{3}$, where

$$
I_{3}=\left[\frac{n_{1}\left(A_{p}+2 / 3\right)-n_{2}}{n}, \frac{n_{1}+n_{2}\left(A_{p}-2 / 3\right)}{n}\right]
$$


Applying inequalities (5.4) and (5.5) we obtain

$$
\frac{n_{1}\left(A_{p}+2 / 3\right)-n_{2}}{n}-\left(A_{p}-\frac{2}{3}\right) \geq \frac{1}{3}-A_{p} \geq 0
$$

and

$$
\left(A_{p}+\frac{2}{3}\right)-\frac{n_{1}+n_{2}\left(A_{p}-2 / 3\right)}{n} \geq A_{p}+\frac{1}{3}>0 .
$$

If $\frac{1}{3}-A_{p}>0$ in (5.8), then the intervals $I_{1}, I_{2}$ and $I_{3}$ are disjoint and Walsh's theorem guarantees that there is a unique zero of $p^{\prime}$ in $I_{3} \subset I$ and therefore $J(p)<\frac{2}{3}$. The equality in (5.1) holds only if $A_{p}=\frac{1}{3}$ in (5.8). Then, from (5.2) and (5.3) we must have $n_{1}=\frac{n}{3}$ and $n_{2}=\frac{2 n}{3}$. Therefore, the only extremal polynomial for $A_{p}>0$ is $p(x)=(x-1)^{\frac{2 n}{3}}(x+1)^{\frac{n}{3}}$.

Case 2: $I$ contains one zero of $p(x)$. Let $t$ be the unique zero of $p(x)$ in I. Let $n_{1}$ and $n_{2}=n-1-n_{1}$ be the numbers of zeros of $p(x)$ in the intervals $I_{1}=\left[-1, A_{p}-2 / 3\right]$ and $I_{2}=\left[A_{p}+2 / 3,1\right]$ respectively. As in the first case, when we fix $t$ and $A_{p}$, we can get bounds for $n_{1}$ and $n_{2}$. If we move all other zeros to the right as far as possible, i.e. put $n_{1}$ zeros at $A_{p}-2 / 3$ and $n_{2}$ zeros at 1 , then the solution of the linear system

$$
\left\{\begin{aligned}
n_{1}\left(A_{p}-2 / 3\right)+n_{2}+t & =n A_{p}, \\
n_{1}+n_{2}+1 & =n
\end{aligned}\right.
$$

gives an upper bound for $n_{1}$ and a lower bound for $n_{2}$. Similarly, if we move all other zeros to the left as far as possible, i.e. put $n_{1}$ zeros at -1 and $n_{2}$ zeros at $A_{p}+2 / 3$ we get a linear system

$$
\left\{\begin{aligned}
-n_{1}+n_{2}\left(A_{p}+2 / 3\right)+t & =n A_{p}, \\
n_{1}+n_{2}+1 & =n
\end{aligned}\right.
$$

that gives a lower bound for $n_{1}$ and an upper bound for $n_{2}$. Combining these two we obtain that the range of $n_{1}$ and $n_{2}$ depending on $A_{p}$ and $t$ is given by

$$
\frac{2 n+3 t+3 A_{p}-2}{5+3 A_{p}} \leq n_{1} \leq 3 \frac{n\left(1-A_{p}\right)+t-1}{5-3 A_{p}}
$$

and

$$
\frac{2 n-3 t+3 A_{p}-2}{5-3 A_{p}} \leq n_{2} \leq 3 \frac{n\left(1+A_{p}\right)-t-1}{5+3 A_{p}} .
$$

We will see that by keeping $t$ and $A_{p}$ fixed and moving the other zeros of $p(x)$ we can get not only the range of $n_{1}$ and $n_{2}$, but also estimate the distance from $A_{p}$ to a nearest zero of $p^{\prime}(x)$. In order to do that we apply so called "Root Dragging Theorem" ([1]) which states that if we move any of the roots of the real-root polynomial to the right (left) then all of the critical points other than multiple roots move to the right (left). Since $t$ is the only zero of $p(x)$ in $I, I$ can contain at most two zeros of $p^{\prime}(x)$ and they must be those that are closest to $t$ from the left and right. Let's call them $w$ and $v, w<t<v$. We will show that at least one of $w$ and $v$ is in $I$. $A_{p}-w$ will attain maximum when we move all zeros as far as possible to the left and so $A_{p}-w \leq A_{p}-w^{\prime}$ where $w^{\prime}$ is the zero of derivative of

$$
(x+1)^{n_{1}}\left(x-\left(A_{p}+2 / 3\right)\right)^{n_{2}}(x-t)
$$


that is closest to $t$ from the left, i.e. $w^{\prime}$ is the leftmost zero of the quadratic equation

$$
\begin{aligned}
& n x^{2}-\left((n-1) t+n_{1}\left(A_{p}+\frac{2}{3}\right)-n_{2}+A_{p}-\frac{1}{3}\right) x \\
& +t\left(n_{1}\left(A_{p}+\frac{2}{3}\right)-n_{2}\right)-A_{p}-\frac{2}{3}=0 .
\end{aligned}
$$

From this and the bounds on $n_{1}$ and $n_{2}$,

$$
A_{p}-w \leq F(t):=\frac{1}{2}\left(2 A_{p}-t+\frac{1}{3}+\sqrt{\left(t+\frac{1}{3}\right)^{2}+\frac{4(t+1)\left(A_{p}-t+\frac{2}{3}\right)}{n}}\right) .
$$

We need to show that $F(t)<2 / 3$ for every $t \in I$. $F(t)-2 / 3$ has at most 2 zeros, which are the zeros of the quadratic function

$$
\begin{aligned}
\bar{F}(t) & :=\frac{4(t+1)\left(A_{p}-t+\frac{2}{3}\right)}{n}+\left(t+\frac{1}{3}\right)^{2}-\left(t+1-2 A_{p}\right)^{2} \\
& =-\frac{4}{n} t^{2}+4 \frac{n+1}{n}\left(A_{p}-\frac{1}{3}\right) t-4\left(A_{p}^{2}-A_{p}-\frac{A_{p}}{n}+2 \frac{n-3}{3 n}\right) .
\end{aligned}
$$

However, $F(t)-2 / 3$ has at most one zero in the interval $I$, since

$$
\begin{aligned}
F\left(A_{p}-\frac{2}{3}\right)-\frac{2}{3} & =\frac{1}{2}\left(A_{p}-\frac{1}{3}+\sqrt{\left(A_{p}-\frac{1}{3}\right)^{2}+\frac{16}{3 n}\left(\frac{1}{3}+A_{p}\right)}\right) \\
& >\frac{1}{2}\left(A_{p}-\frac{1}{3}+\left|A_{p}-\frac{1}{3}\right|\right)=0
\end{aligned}
$$

and

$$
F\left(A_{p}+\frac{2}{3}\right)-\frac{2}{3}=\frac{1}{2}\left(A_{p}+\left|A_{p}+1\right|-\frac{5}{3}\right)=A_{p}-\frac{1}{3} \leq 0 .
$$

Also the leading coefficient in $\bar{F}(t)$ is negative and

$$
\bar{F}\left(A_{p}-\frac{2}{3}\right)=\frac{16}{3 n}\left(\frac{1}{3}+A_{p}\right)>0 \text { and } \bar{F}\left(A_{p}+\frac{2}{3}\right)=\frac{16}{3}\left(A_{p}-\frac{1}{3}\right) \leq 0,
$$

so the only zero of $F(t)-2 / 3$ in $I$ can be $\eta$, the rightmost zero of $\bar{F}(t)$ and $F(t) \leq \frac{2}{3}$ in $I$ if $t \geq \eta$. Also $v-A_{p}$ will attain maximum when we move all zeros as far as possible to the right and $v-A_{p} \leq v^{\prime}-A_{p}$ where $v^{\prime}$ is the zero of derivative of

$$
\left(x-\left(A_{p}-2 / 3\right)\right)^{n_{1}}(x-1)^{n_{2}}(x-t)
$$

that is closest to $t$ from the right, i.e. $v^{\prime}$ is the rightmost zero of the quadratic equation

$$
\begin{aligned}
& n x^{2}-\left((n-1) t+n_{2}\left(A_{p}-\frac{2}{3}\right)+n_{1}+A_{p}+\frac{1}{3}\right) x \\
& +t\left(n_{2}\left(A_{p}-\frac{2}{3}\right)+n_{1}\right)+A_{p}-\frac{2}{3}=0 .
\end{aligned}
$$


From this and the bounds on $n_{1}$ and $n_{2}$,

$$
v-A_{p} \leq G(t):=\frac{1}{2}\left(-2 A_{p}+t+\frac{1}{3}+\sqrt{\left(-t+\frac{1}{3}\right)^{2}+\frac{4(-t+1)\left(-A_{p}+t+\frac{2}{3}\right)}{n}}\right) .
$$

We need to show that $G(t)<2 / 3$ for every $t \in I$. $G(t)-2 / 3$ has at most 2 zeros, which are the zeros of the quadratic function

$$
\begin{aligned}
\bar{G}(t) & :=\frac{4(-t+1)\left(-A_{p}+t+\frac{2}{3}\right)}{n}+\left(-t+\frac{1}{3}\right)^{2}-\left(t-1-2 A_{p}\right)^{2} \\
& =-\frac{4}{n} t^{2}+4 \frac{n+1}{n}\left(A_{p}+\frac{1}{3}\right) t-4\left(A_{p}^{2}+A_{p}+\frac{A_{p}}{n}+2 \frac{n-3}{3 n}\right) .
\end{aligned}
$$

However, $G(t)-2 / 3$ has at most one zero in the interval $I$, because

$$
G\left(A_{p}-\frac{2}{3}\right)-\frac{2}{3}=\frac{1}{2}\left(-A_{p}+\left|A_{p}-1\right|-\frac{5}{3}\right)=-A_{p}-\frac{1}{3}<0
$$

and

$$
\begin{aligned}
G\left(A_{p}+\frac{2}{3}\right)-\frac{2}{3} & =\frac{1}{2}\left(-A_{p}-\frac{1}{3}+\sqrt{\left(A_{p}+\frac{1}{3}\right)^{2}+\frac{16}{3 n}\left(\frac{1}{3}-A_{p}\right)}\right) \\
& \geq \frac{1}{2}\left(-A_{p}-\frac{1}{3}+\left|A_{p}+\frac{1}{3}\right|\right)=0 .
\end{aligned}
$$

Also the leading coefficient in $\bar{G}(t)$ is negative and

$$
\bar{G}\left(A_{p}-\frac{2}{3}\right)=-\frac{16}{3}\left(\frac{1}{3}+A_{p}\right)<0 \text { and } \bar{G}\left(A_{p}+\frac{2}{3}\right)=\frac{16}{3 n}\left(\frac{1}{3}-A_{p}\right) \geq 0,
$$

so the only zero of $G(t)-2 / 3$ in $I$ can be $\xi$, the leftmost zero of $\bar{G}(t)$ and $G(t) \leq \frac{2}{3}$ in $I$ if $t \leq \xi$. We want to show that $\xi \geq \eta$. The equation $\bar{F}(t)=\bar{G}(t)$ has the unique solution $t=3 A_{p}$ so it's enough to show that $\bar{F}\left(3 A_{p}\right)=\bar{G}\left(3 A_{p}\right)<0$. But

$$
\bar{F}\left(3 A_{p}\right)=8 \frac{n-3}{n}\left(A_{p}-\frac{1}{3}\right)\left(A_{p}+\frac{1}{3}\right) \leq 0 \text { for } 0 \leq A_{p} \leq \frac{1}{3}
$$

and equality is attained only if $A_{p}=\frac{1}{3}$ and $t=1$, but this is case $I$, and we must have $p(x)=(x-1)^{\frac{2 n}{3}}(x+1)^{\frac{n}{3}}$ with $J(p)=\frac{2}{3}$. Otherwise $\xi>\eta$ and either $\xi$ or $\eta$ lie in $I$ and $J(p)<\frac{2}{3}$. If $A_{p}<0$ the above argument applied to polynomial $p(-x)$ shows that $J(p)=\frac{2}{3}$ only if $p(x)=(x+1)^{\frac{2 n}{3}}(x-1)^{\frac{n}{3}}$.

\section{REFERENCES}

[1] B. Anderson, Polynomial Root Dragging, Amer. Math. Monthly 100 (1993), 864-866. MR 94i:26006

[2] M. Marden, Conjectures on the critical points of a polynomial, Amer. Math. Monthly 90 (1983), 267-276. MR 84e:30007

[3] M. Marden, Geometry of Polynomials, Math. Surveys 3, Amer. Math. Soc., Providence, R.I. 1966. MR 37:1562

[4] M.J. Miller, Maximal polynomials and the Ilieff-Sendov conjecture, Trans. Amer. Math. Soc. 321 (1990), 285-303. MR 90m:30007 
[5] M.J. Miller, Continuous independence and the Ilieff-Sendov conjecture, Proc. Amer. Math. Soc. 115 (1992), 79-83. MR 92h:30012

[6] G.V. Milovanovic, D.S. Mitrinovic, and Th.M. Rassias, Topics in polynomials : extremal problems, inequalities, zeros, World Scientific, River Edge, NJ, 1994. MR 95a:30009

[7] D. Phelps and R.S. Rodriguez, Some properties of extremal polynomials for the Ilieff conjecture, Kodai Math. Sem. Report 24 (1972), 172-175. MR 46:3753

[8] R. M. Robinson, On the span of derivatives of polynomials, Amer. Math. Monthly 71 (1964), 504-508. MR 28:5135

[9] R. M. Robinson, Algebraic equations with span less than 4, Math. Comp. 18 (1964), 547-559. MR 29:6624

[10] G. Schmeisser, On Ilieff's Conjecture, Math. Z. 156 (1977), 165-173. MR 58:6182

Department of Mathematics and Computer Science, Kent State University, Kent, Оніо 44242

Current address: Summit Systems, Inc., 22 Cortland St., New York, New York 10007

E-mail address: piotr-pawlowski@summithq.com 